OPEN ACCESS

Edited by: Amaresh Chandra Panda, Institute of Life Sciences (ILS), India

Reviewed by: Isabel Chillón European Molecular Biology Laboratory Grenoble, France Piyush Khandelia Birla Institute of Technology and Science, India

*Correspondence: Zi-Bing Jin jinzb502@ccmu.edu.cn; jinzibing@foxmail.com

Specialty section: This article was submitted to

Protein and RNA Networks, a section of the journal Frontiers in Molecular Biosciences

Received: 15 November 2020

Accepted: 25 February 2021

Published: 19 March 2021

Citation:

Li M-L, Wang W and Jin Z-B (2021) Circular RNAs in the Central Nervous System.

Front. Mol. Biosci. 8:629593. doi: 10.3389/fmolb.2021.629593

\section{Circular RNAs in the Central Nervous System}

\author{
Meng-Lan Li, Wen Wang and Zi-Bing Jin* \\ Beijing Institute of Ophthalmology, Beijing Tongren Eye Center, Beijing Tongren Hospital, Capital Medical University, Beijing \\ Ophthalmology \& Visual Sciences Key Laboratory, Beijing, China
}

Circular RNAs (circRNAs) are endogenous single-stranded RNAs characterized by covalently closed loop structures with neither $5^{\prime}$ to $3^{\prime}$ polarity nor poly(A) tails. They are generated most commonly from back-splicing of protein-coding exons. CircRNAs have a tissue-specific distribution and are evolutionarily conserved, and many circRNAs play important biological functions by combining with microRNAs and proteins to regulate protein functions and their own translation. Numerous studies have shown that circRNAs are enriched in the central nervous system (CNS) and play an important role in the development and maintenance of homeostasis. Correspondingly, they also play an important role in the occurrence and progression of CNS diseases. In this review, we highlight the current state of circRNA biogenesis, properties, function and the crucial roles they play in the CNS.

Keywords: circular RNA, review (article), function, disease, central nervous system

\section{INTRODUCTION}

For many years, although proven to be present, circular RNAs (circRNAs) were overlooked as byproducts of splicing errors (Sanger et al., 1976; Barrett and Salzman, 2016). However, major recent studies have discovered that circRNAs have essential functions and play a novel regulatory role, especially in the nervous system (Memczak et al., 2013), prompting interest from an increasing number of investigators from various fields. Unlike messenger RNAs (mRNAs), circRNAs are mostly derived from different regions of gene loci in eukaryotes through a non-canonical splicing process called "back-splicing." During back-splicing, the downstream $5^{\prime}$ splice site is covalently bonded to an upstream $3^{\prime}$ splice site in a reversed orientation. Due to the lack of $5^{\prime}-3^{\prime}$ polarity and a polyadenylated tail, circRNAs are much more insusceptible than linear RNAs to degradation by exonuclease RNase R. CircRNAs exert their action mainly by acting as a miRNA sponge and functioning through a competing endogenous RNA (ceRNA) mechanism (Hansen et al., 2013; Memczak et al., 2013), as well as acting as protein sponges or translating proteins (Legnini et al., 2017). Recent genome-wide profiling of circRNAs has shown that numerous circRNAs are widely and dynamically expressed in the nervous system. Furthermore, their expression is dramatically increased in the brain during the aging of multiple organisms (Knupp and Miura, 2018). In addition, circRNAs participate in many processes of neurological diseases. For instance, circTTBK2 and circPCMTD1 act as sponges of miR-224-5p to promote glioma progression (Zheng et al., 2017, 2019). As an extension of the brain and a part of the central nervous system (CNS), the retina is another option for investigators to study enigmas of circRNAs. It has been demonstrated that circRNAs are abundant in the retina and play a role in biogenesis and various biological functions (Wang et al., 2018a; Chen et al., 2020c). 


\section{BIOGENESIS, PROPERTIES, AND FUNCTIONS OF CIRCRNAS}

\section{Biogenesis}

Circular RNAs can be generated from different gene loci, such as coding and non-coding exons, introns, both exons and introns, or antisense or intergenic sequences. Due to their different origins, they have different names, such as exonic circRNA (or ecircRNA), intronic circRNA (or ciRNA), and exon-intron circRNA (or ElciRNA) (Figure 1; Zhang et al., 2013; Guo et al., 2014; Li et al., 2015). These circRNAs can be generated in different manners. (1) They can be generated from intronic lariat precursors that escape from the debranching step of canonical linear splicing (Figure 1A; Zhang et al., 2013). A pre-mRNA can generate linear RNA, lariat introns, Y-structure introns from trans-splicing, and circular exons through exon skipping (Suzuki et al., 2006). In canonical splicing, a lariat intron is generated after the splicing of the linear pre-mRNA and isolated from the ultimate mRNA product. This excised lariat undergoes internal back-splicing. Then, lariats that escape from debranching can lead to the formation of ciRNAs (Zhang et al., 2013; Eger et al., 2018). (2) Lariat formation during exon skipping (Figure 1B; Kelly et al., 2015; Petkovic and Muller, 2015). During alternative splicing, an exon-skipping event occurs and creates an exon-containing lariat formation, intronic lariat formation and mRNA with skipped exons (Zaphiropoulos, 1997; Barrett et al., 2015). The exoncontaining lariat creates ecircRNAs when undergoing internal back-splicing (Kelly et al., 2015; Petkovic and Muller, 2015). However, not all of the exon-containing lariats will generate circular RNA despite the correlation between exon-skipping and circular RNA formation (Barrett et al., 2015; Kelly et al., 2015). And the smaller skipped exons are less prone to circularize than those in large size (Barrett et al., 2015). (3) Looped by base pairing between inverted repeat elements (such as Alu elements) (Jeck et al., 2013; Ivanov et al., 2015) or RNA-binding proteins (RBPs) (Figure 1C; Errichelli et al., 2017). Looping of the intron sequences flanking the downstream splice-donor site and the upstream splice-acceptor site brings these sites into close proximity (Ivanov et al., 2015). RBPs can bind two flanking introns together to promote the formation of circular structures; then, introns will be removed to form junction sites. For instance, muscleblind protein (MBL) provokes circMbl biogenesis in the form of RBPs, resulting in competition between circMbl and its linear transcript (Ashwal-Fluss et al., 2014). Similarly, the immune factors NF90/NF110 also promote circRNA production in the nucleus as RBPs ( $\mathrm{Li}$ et al., 2017). In addition, some cisregulatory elements and trans-acting factors participate in backsplicing, such as the spliceosome assembly (Zhang et al., 2016). Although studies have illustrated these mechanisms of circRNA biogenesis, this process has not been fully elucidated.

\section{Properties}

As circular structure, circRNAs have some common characteristics. First, circRNAs are prevalent across species and evolutionarily conserved. They can be detected in many species, from plants to animals and from Caenorhabditis elegans to humans (Memczak et al., 2013; Sun et al., 2019). With a strict definition, investigators found that 4,522 of 15,849 mouse circRNAs were conserved in humans (Rybak-Wolf et al., 2015). For example, the circTulp4 isoform is derived from homologous exons in humans and mice (Rybak-Wolf et al., 2015). Second, circRNAs have tissue/developmentalstage-specific expression. For instance, circCdrlas is abundantly expressed in the nervous system, whereas it is barely detected in non-neuronal tissues (Memczak et al., 2013; Chen et al., 2020b). In addition, circRNAs are dynamically expressed during development. In the mouse hippocampus, circRNAs derived from protein coding gene loci with synapse-related functions were observed to be upregulated from E18 to P30 [You et al. (2015) profiled four stages: embryonic (E18), early postnatal (P1), the beginning of synapse formation (P10), and late postnatal (P30)]. In contrast, those produced from gene loci without any function were downregulated. Third, circRNAs are much more resistant than linear RNAs. Due to the closed loop structure, circRNAs are stable and can resist degradation from RNase $\mathrm{R}$, which indicates why circRNAs can accumulate in cells for a long time (You et al., 2015). Fourth, circRNAs have incredible diversity. Different circRNAs can consist of one or more exons or even no exons (intronic circRNA), but they principally contain 2-5 exons (Rybak-Wolf et al., 2015). The length of circRNAs ranges from $100 \mathrm{bp}$ to $4 \mathrm{~kb}$ (Salzman et al., 2013).

\section{Biological Function}

As a novel type of RNA, circRNAs have been proven to play various roles in biological processes. First, individual circRNAs have been posited to function as miRNA or RNA-binding protein sponges (Figure 2A). A famous circRNA, ciRS-7, also known as circCdrlas, has more than 70 putative binding sites for miR-7, allowing multiple interactions. Knockdown of circCdrlas decreased the expression of miR-7 target genes, whereas knockout of circCdrlas downregulated miR-7 (Hansen et al., 2013; Memczak et al., 2013; Piwecka et al., 2017). Regarding the combination of RBP, the tumor suppressor gene Foxo3 can produce circFoxo3, and circFoxo3 regulates cell cycle progression by binding to cell division protein kinase 2 (CDK2) and cyclin-dependent kinase inhibitor 1 (p21) (Diallo et al., 2019). Second, circRNA can be translated in cap-independent manners. Under stress conditions, circRNA can use its internal ribosome entry site (IRES) to recruit ribosomes to an internal position of circRNA. For example, circMbl shares the same start codon as the linear mRNA and can be found with increasing IRES activity. The proteins detected by mass spectrometry also provide important evidence (Pamudurti et al., 2017) (Figure 2B left). The second mechanism for circRNA translation is the recruitment of eukaryotic initiation factor 3 (eIF3) by methylated adenosine residues in the form of N6-methyladenosines (m6A) in the $5^{\prime}$ untranslated region $\left(5^{\prime} \mathrm{UTR}\right)$ for translation into small polypeptides (Diallo et al., 2019; Zhou et al., 2021) (Figure 2B right). Third, circRNA can participate in transcriptional regulation by interacting with RNA polymerase II (RNA pol II) and other snRNP partners. Experiments have shown that 


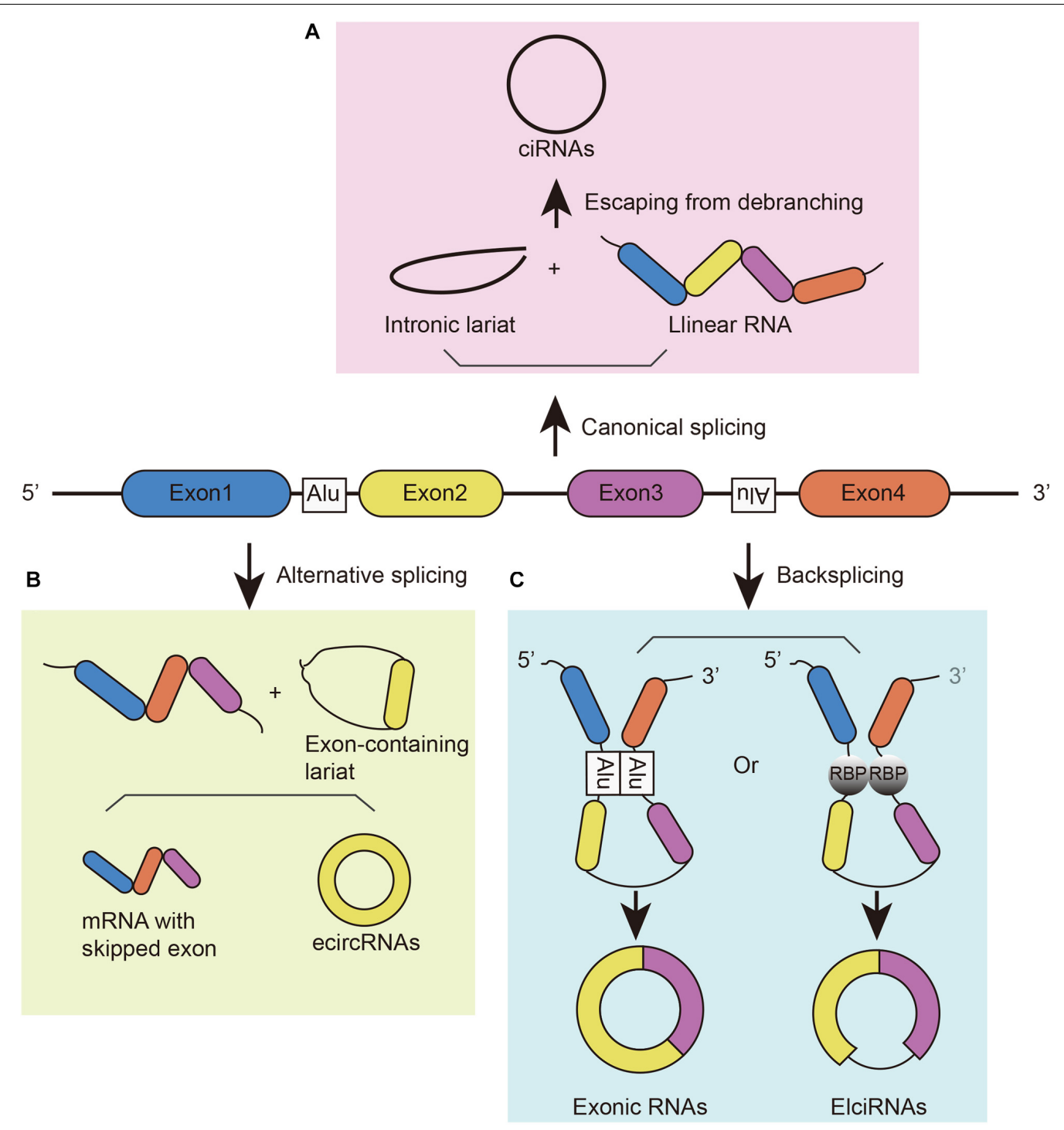

FIGURE 1 | The biogenesis of circRNAs. (A) CiRNAs are generated from intronic lariat precursors that escape from the debranching step of canonical linear splicing (B) EcircRNAs can be generated from exon-containing lariats created by an exon-skipping event during linear splicing. (C) CircRNAs can be generated from back-splicing mediated by inverted repeat elements and trans-acting RNA binding proteins.

circEIF3J and circPAIP2 can regulate the transcription of their parental genes through this mechanism (Li et al., 2015; Figure 2C).

\section{DISTRIBUTION, \\ DEVELOPMENTAL-STAGE-SPECIFIC EXPRESSION PROFILE, AND AGE-RELATED ACCUMULATION OF CIRCRNAS IN THE NERVOUS SYSTEM}

\section{Distribution}

Circular RNAs has been shown to be tissue-specific. We compared the number of circRNAs detected in human tissues in the TSCD database ${ }^{1}$ and found that circRNAs are highly enriched in the brain (Figure 3; Xia et al., 2017). It was observed that the brain had a dominant role not only in the number of circRNAs but also in the frequency of circRNA hosting genes (approximately $20 \%$ of brain protein-coding genes produce circRNAs) (You et al., 2015). Another study reached a similar conclusion by comparing the human frontal cortex, thyroid gland, liver, and muscle (Rybak-Wolf et al., 2015). The enriched circRNAs are not uniformly distributed throughout the nervous system; it has been proven that they vary in different brain areas (Rybak-Wolf et al., 2015). A comparison of the circRNA expression of areas in the human and mouse brain showed that circRNAs were mostly enriched in the forebrain in mice

\footnotetext{
${ }^{1}$ http://gb.whu.edu.cn/TSCD
} 


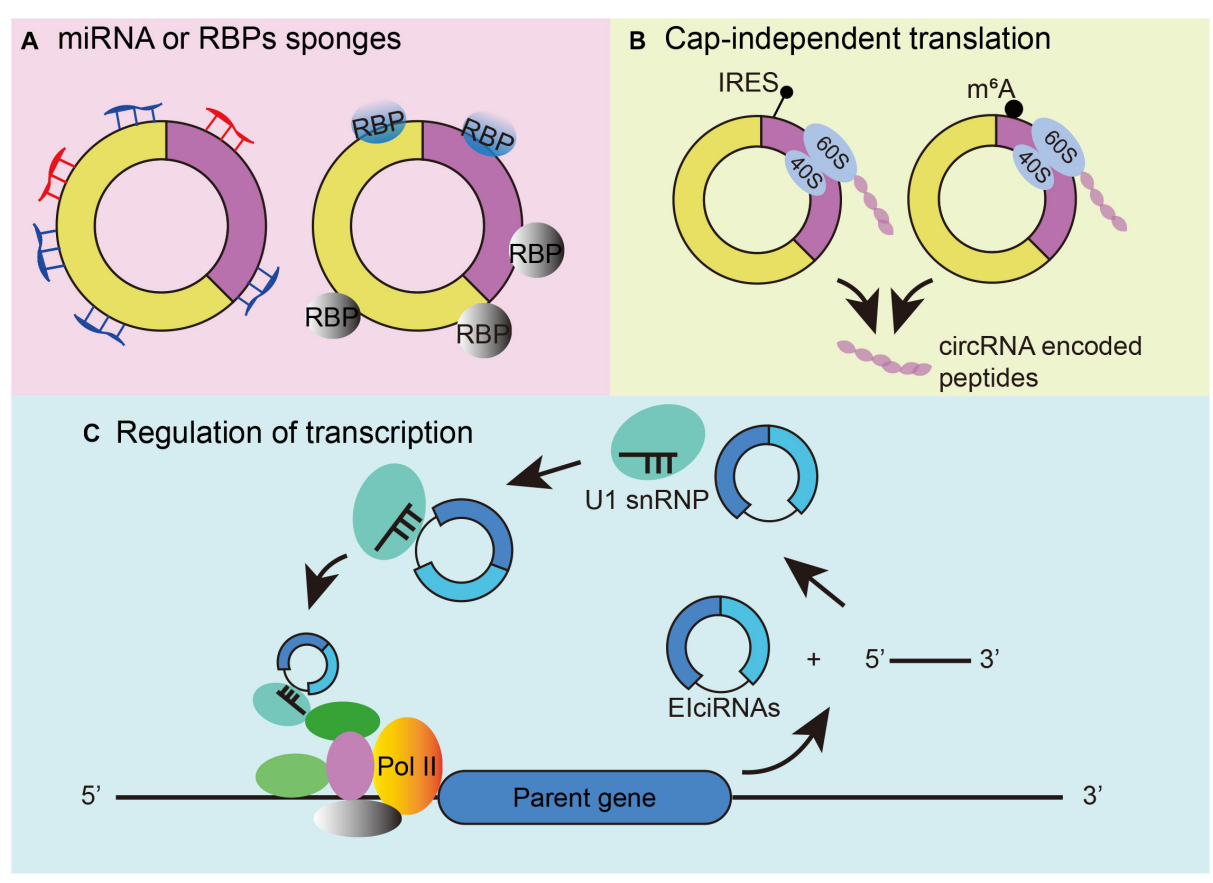

FIGURE 2 | Mechanisms of circRNA functions. (A) CircRNAs can function as microRNA and RBP sponges. (B) CircRNA cap-independent translation mechanism: IRES-driven circRNA translation (left) and m6A-driven circRNA translation (right). (C) Regulation of transcription initiation by ElciRNAs.

and that the prefrontal cortex (PFC) had greater expression than the hippocampus (HC). Investigators assessed genomewide expression of circRNAs in the HC and PFC of the mouse brain (Chen et al., 2018) and found an opposite result to that of Rybak-Wolf's research; namely, circRNA expression in the $\mathrm{HC}$ was greater than that in the PFC. This finding may have occurred because Chen et al. (2018) chose data from the GEO database, while Rybak-Wolf et al. (2015) detected and analyzed these molecules on their own. Another reason may be the sample differences. However, both studies demonstrated the potential function of circRNAs in essential neuronal activities. Afterward, investigators further explored the exact enrichment localization of circRNAs in cells (You et al., 2015). Gene Ontology analysis indicated that circRNAs in the brain are mostly derived from several groups of genes related to synaptic function. Thus, highresolution in situ hybridization (ISH) showed that localization of circRNAs was found in both the cell body and the dendrites of neurons (You et al., 2015). Furthermore, it was found that circRNAs were more abundant in synaptoneurosomes than whole-brain lysate and cytoplasm based on all expression cutoffs when they were normalized to host gene expression (Rybak-Wolf et al., 2015). The localization of circRNAs in the synaptic neuropil suggests that these molecules may play a role in the regulation of gene expression required for synaptic plasticity.

\section{Developmental-Stage-Specific Expression Profile}

It has been proven that circRNAs are expressed in a developmental-stage-specific manner. During the maturation of primary neurons, most circRNAs (1,926 circRNAs) were found to be upregulated and only a few were downregulated (797 circRNAs) in the mouse brain (Rybak-Wolf et al., 2015). Investigation of Drosophila showed that the expression of circRNAs in neurons was increased throughout life (Westholm et al., 2014). During porcine embryonic brain development (E23, E42, E60, E80, E100, and E115) (Venø et al., 2015), circRNAs were increased from E23 to E60 and reached their peak at E60. Then, expression declined drastically with continuing reduction until E115. These implicit circRNAs may function at specific developmental periods and are important for neuronal function. In addition, investigators found that when compared with mRNA of their host gene, circRNAs were not expressed in the same way. The negative correlation between gene expression and the logarithm of the circular-to-linear ratio (CLR) indicates the independent function of circRNAs (Rybak-Wolf et al., 2015). The expression of circRNAs in the retina was also explored. It was found that many circRNAs were upregulated or downregulated during immature rat retinal development in P3, P7, and P12 (Han et al., 2017). Work from Chen et al. (2020c) in the mouse retina obtained a similar conclusion and indicated that the expression patterns of circRNAs differed from linear transcripts from the same host gene. The developmental stage-specific expression profile of circRNA suggests its important regulatory function in the development and differentiation of the nervous system. The data from the retina are not completely consistent with those from the brain; thus, further research is needed.

\section{Age-Related Accumulation}

In addition to the early stages of development, investigators are also interested in the expression of circRNAs in the aging nervous 


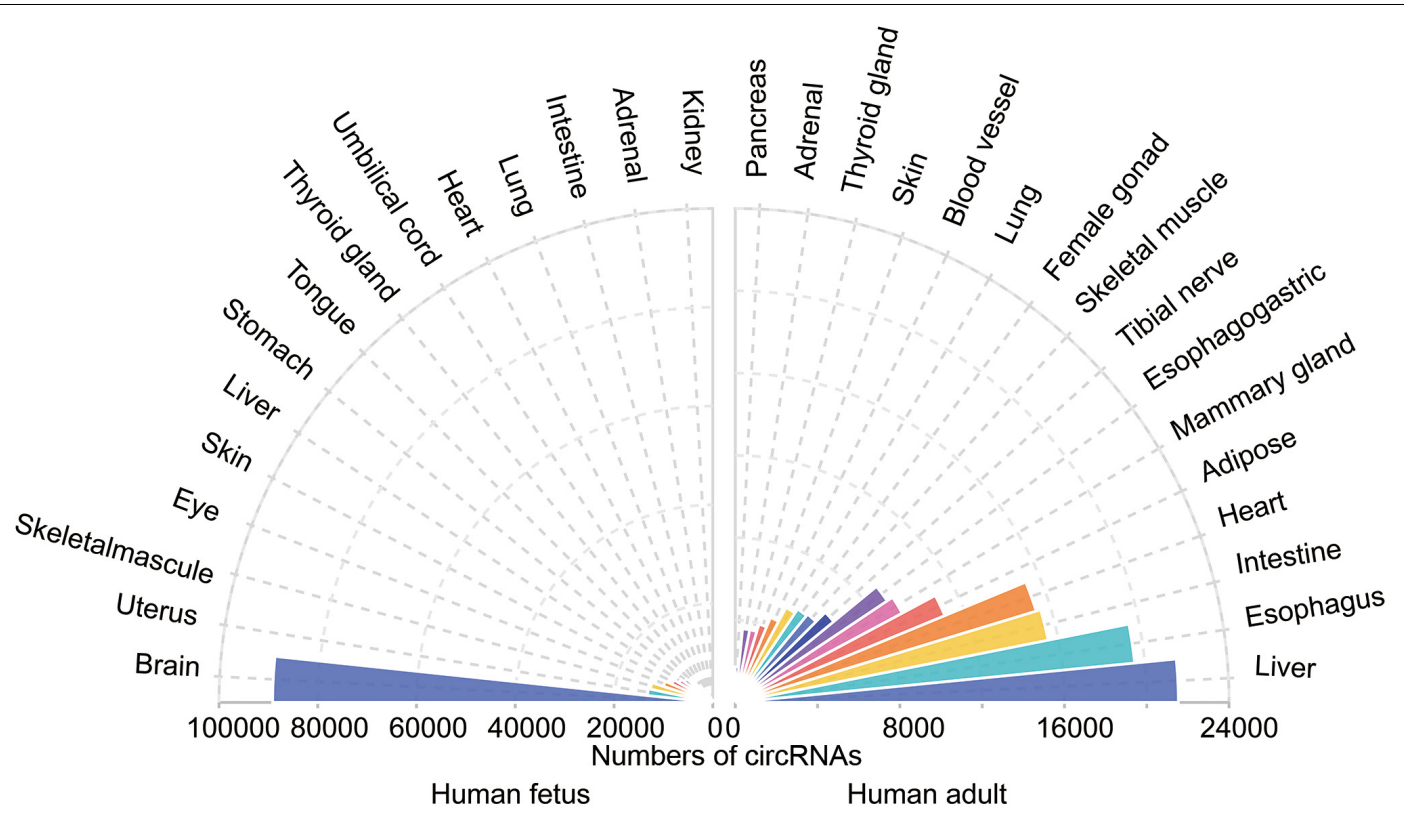

FIGURE 3 | The tissue-specific circRNAs expressed in human tissues. The colorful triangles represent the numbers of circRNAs detected in different human tissues (left, 15 human fetal tissues; right, 15 human adult tissues), respectively. CircRNAs are most abundant in the human fetus brain compared with other tissues.

system. To date, several studies have shown that age-related circRNA accumulation exists in various animals (Westholm et al., 2014; Gruner et al., 2016; Cortes-Lopez et al., 2018; Chen et al., 2019). Drosophila melanogaster RNA-Seq libraries reveal this phenomenon for the first time. Westholm et al. found that the expression of 262 circRNAs was significantly upregulated in 20-day heads versus 1-day heads (Westholm et al., 2014). The circRNAs identified in the cortex, HC and heart of 1- and 22-month-old mice were analyzed (Gruner et al., 2016). The study revealed that circRNAs showed significant upregulation in elderly cortex and hippocampal samples but not in the heart. Moreover, investigators performed additional validations using the cortex from an intermediate age of 6 months (Gruner et al., 2016). They found that half of the circRNAs were significantly increased between 1 and 6 months, and all detected circRNAs were significantly increased between 6 and 22 months. This result revealed that the age-dependent accumulation was progressive. In addition, aging-related accumulation of circRNAs was found in C. elegans and porcine brain (Cortes-Lopez et al., 2018; Chen et al., 2019). The latest research on rhesus monkeys showed that 11 circRNAs and host mRNAs are involved in regulating brain aging (Xu et al., 2020). One of them, circGRIA1, can regulate agerelated synaptic plasticity by negatively regulating its host gene in the nucleus. Interestingly, the accumulation of circGRIA1 is also male-biased, which warrants further study.

The above results revealed that circRNAs are highly expressed in the nervous system. The uneven distribution of circRNAs in different brain regions indicates that they have potential functions in spatial learning and memory. The age-related accumulation of circRNAs suggests that they may contribute to neuronal aging and age-related diseases such as Alzheimer's disease (AD) (Lukiw and Circular, 2013) and age-related macular degeneration (AMD) (Jin et al., 2019; Chen et al., 2020a). Current studies show some evidences in these diseases but still requires further research.

\section{CIRCRNAS IN NEUROLOGICAL DISEASES}

With such abundant expression in the nervous system, circRNAs play important roles in neurological diseases, such as $\mathrm{AD}$, Parkinson's disease (PD), and immune-mediated demyelinating diseases (Ghosal et al., 2013; Lukiw and Circular, 2013; Zhao et al., 2016; He et al., 2019). Briefly, we summarized some circRNAs involved in neurological diseases (Table 1) and three representative ones were chosen for detailed depiction (Figure 4).

\section{AD}

The miR-7 circRNA system was shown to be dysregulated in the hippocampal CA1 region of sporadic AD patients by Northern blot hybridization techniques and the circularitysensitive circRNA probe RNase R (Lukiw and Circular, 2013). It has been demonstrated that ubiquitin protein ligase A (UBE2A) is a target downstream gene of miR-7. UBE2A is the core effector of the ubiquitin $26 \mathrm{~S}$ proteasome system, which acts by proteolysis to remove amyloid peptides. The expression of miR-7 is significantly increased in the brains of sporadic $\mathrm{AD}$ patients, which may be related to the downregulation of circCdrlas expression (Zhao et al., 2016). In addition, circCdrlas can promote the degradation of APP and BACE1 via the proteasome and lysosome (Shi et al., 2017; Figure 4A). Furthermore, the atlas of cortical circular RNA expression in $\mathrm{AD}$ and normal patients' brains showed that circRNA expression 
TABLE 1 | CircRNAs in neurological diseases.

\begin{tabular}{|c|c|c|c|c|c|}
\hline Disease/Model & Host & & CircRNAs & Mechanism & Reference \\
\hline \multirow[t]{3}{*}{$A D$} & & Human & circCDR1as & As miR-7 sponge & $\begin{array}{l}\text { Zhao et al., 2016; Shi } \\
\text { et al., } 2017\end{array}$ \\
\hline & & Human & circHOMER1 & As miR-651 sponge & Dube et al., 2019 \\
\hline & & Human & circCORO1C & As miR-105 sponge & Dube et al., 2019 \\
\hline \multirow[t]{2}{*}{$A D$} & $\mathrm{HN}$ cell & Human & circHDAC9 & $\begin{array}{l}\text { Alleviated } A \beta 42 \text {-induced HN cell neurotoxicity } \\
\text { via miR-142-5p }\end{array}$ & Zhang Y. et al., 2020 \\
\hline & cellular AD model & Rat & circ 0000950 & $\begin{array}{l}\text { circ } 0000950 \text { enhanced neuron apoptosis and } \\
\text { inflammatory response in } A D \text { through acting as } \\
\text { a miR-103 sponge }\end{array}$ & Yang et al., 2019 \\
\hline \multirow[t]{2}{*}{ PD } & & Human & circCDR1as & As miR-7 sponge & Ghosal et al., 2013 \\
\hline & & Mouse & circDLGAP4 & miR-134-5p/CREB pathway & Feng et al., 2020 \\
\hline \multirow{2}{*}{\multicolumn{2}{|c|}{ Immune-mediated demyelinating disease }} & Human & hsa circ 0087862 & Biomarker & He et al., 2019 \\
\hline & & & hsa circ 0012077 & & \\
\hline \multirow[t]{4}{*}{ CNS injury } & $\begin{array}{l}\text { HT22 cells with oxygen-glucose } \\
\text { deprivation/reoxygenation } \\
\text { (OGD/R) }\end{array}$ & Mouse & mmu-circRNA-015947 & $\begin{array}{l}\text { mmu-miR-188-3p, mmu-miR-329-5p, } \\
\text { mmu-miR-3057-3p, mmu-miR-5098, } \\
\text { mmu-miR-683 sponge }\end{array}$ & Lin et al., 2016 \\
\hline & $\begin{array}{l}\text { Microglia-induced hippocampal } \\
\text { neuronal apoptosis }\end{array}$ & Rat & circPTK2 & MiR-29b-SOCS-1-JAK2/STAT3-IL-1 $\beta$ pathway & Wang et al., 2019 \\
\hline & Traumatic injury & Rat & circ-Spidr & PI3K-Akt signaling pathway & Mao et al., 2019a \\
\hline & Nerve crush model & Rat & circ-Ankib1 & $m i R-423-5 p$, miR-485-5p, and miR-666-3p & Mao et al., 2019b \\
\hline \multirow[t]{4}{*}{ Retinal disease } & $\mathrm{RB}$ & Human & hsa circ 0001649 & AKT/mTOR signaling pathway & Xing et al., 2018 \\
\hline & AMD/RPE cell line & Human & circNR3C1 & circNR3C1-miR-3 82-5p-PTEN network & Chen et al., 2020a \\
\hline & Rat model of glaucoma & Rat & circ-ZRANB1 & circ-ZRANB1/miR-217/RUNX2 network & Wang et al., 2018b \\
\hline & Rat model of glaucoma & Rat & cZNF609 & As miR-615 sponge & Wang et al., 2018a \\
\hline \multirow[t]{5}{*}{ Glioma } & Glioma cell lines & Human & circ-ZNF264 & As miR-4493 sponge & Zhang et al., 2019 \\
\hline & & Human & circPCMTD1 & As miR-224-5p sponge & Zheng et al., 2019 \\
\hline & $\begin{array}{l}\text { Human tissue sample and cell } \\
\text { line }\end{array}$ & Human & circ-ТTBK2 & As miR-217 sponge & Zheng et al., 2017 \\
\hline & Human brain sample & Human & circ-FBXW7 & Encode protein & Yang et al., 2018 \\
\hline & $\begin{array}{l}\text { Human tissue sample and cell } \\
\text { line }\end{array}$ & Human & circPOSTN & CircPOSTN/miR-361-5p/TPX2 axis & Long et al., 2020 \\
\hline
\end{tabular}

AD, Alzheimer's disease; PD, Parkinson's disease; CNS, central nervous system; RB, retinoblastoma; AMD, Age-related macular degeneration.

levels are significantly correlated with both neuropathological and clinical measures of $\mathrm{AD}$ severity (Dube et al., 2019). In this study, circHOMER1 was most significantly correlated with $\mathrm{AD}$ and may be involved in $\mathrm{AD}$ as a sponge of miR651, which regulates the AD-related genes PSEN1 and PSEN2 (Agarwal et al., 2015). Recent in vitro studies found additional circRNAs involved in $\mathrm{AD}$. In A $\beta 42$-treated $\mathrm{HN}$ cells, circHDAC9 overexpression can promote cell viability and repress cell apoptosis and inflammation via sponging miR-142-5p (Zhang N. et al., 2020). In a rat cellular AD model, circ_0000950 was found to promote neuronal apoptosis and the inflammatory response in $\mathrm{AD}$ via sponging miR-103 (Yang et al., 2019). The mechanism of circRNAs in $\mathrm{AD}$ remains to be studied in the future, which will provide a new direction for the treatment of $\mathrm{AD}$.

\section{PD}

MiR-7 also plays a role in PD by regulating alpha-synuclein (Ghosal et al., 2013). Alpha-synuclein is overexpressed with the development of PD and plays an essential role in PD. Overexpression of miR-7 can reduce the level of alpha-synuclein (Doxakis, 2010). Considering the interaction between mir-7 and circCdrlas, circCdrlas may participate in the PD pathological process by acting as a sponge. In addition, investigators found that circDLGAP4 had neuroprotective effects by modulating the miR-134$5 \mathrm{p} / \mathrm{CREB}$ pathway in a PD mouse model (Feng et al., 2020), although the mechanism underlying circRNAs in $\mathrm{PD}$ is still unclear.

\section{Immune-Mediated Demyelinating Disease}

CircRNA expression was dysregulated in cerebrospinal fluid from patients with immune-mediated demyelinating disease compared with that of healthy controls (2,364 were upregulated and 2,730 were downregulated) (He et al., 2019). The enrichment analysis of GO and KEGG showed that these circRNAs are most likely to participate 


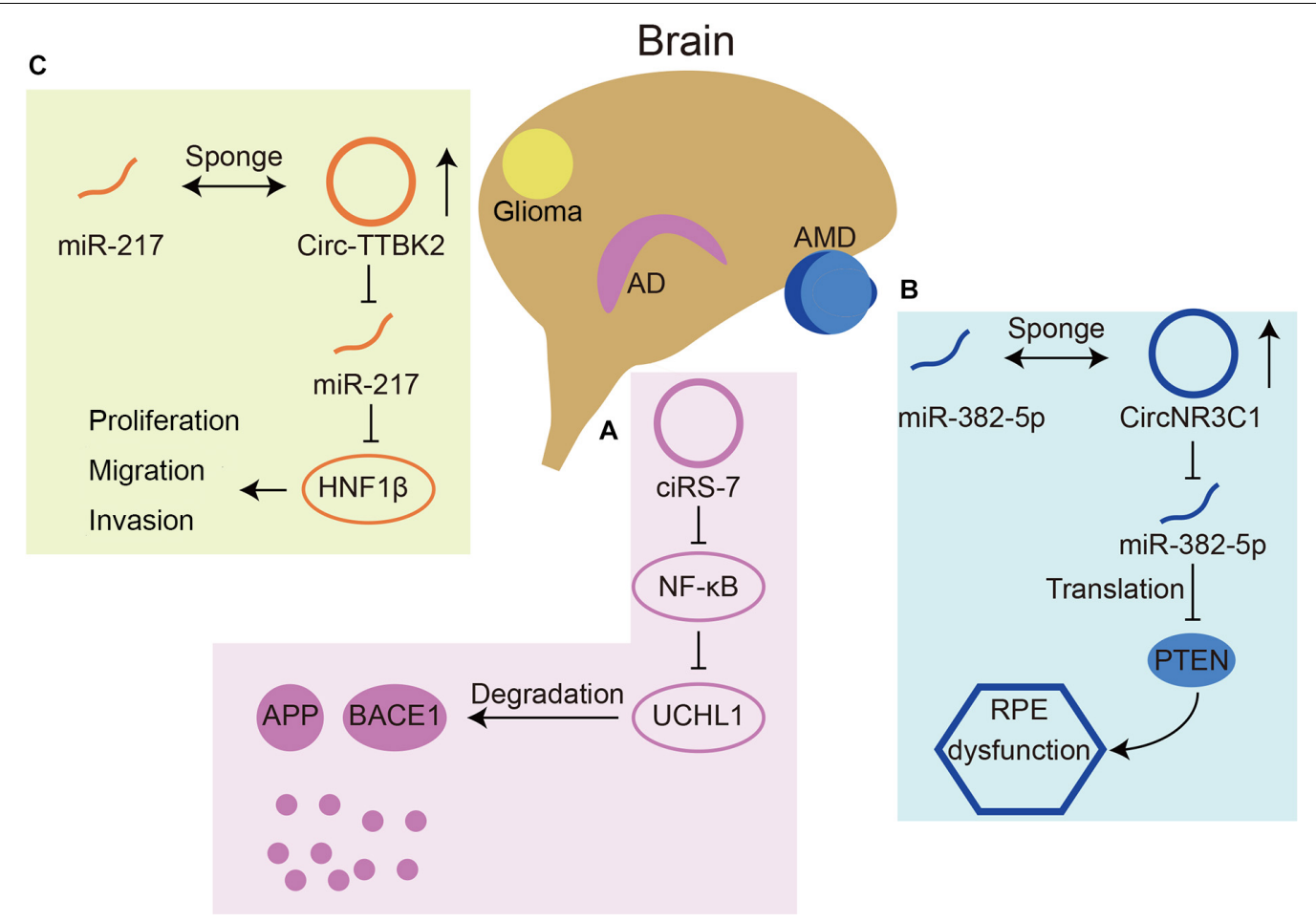

FIGURE 4 | Three circRNAs that play roles in neurological diseases. (A) CiRS-7(also named circCDR1as) can inhibit translation of NF-kB, resulting in upregulated expression of UCHL1. UCHL1 can promote the degradation of APP and BACE1 in AD. (B) CircNR3C1 acts as a miR-382-5p sponge, which can inhibit translation of an AMD related gene PTEN. (C) Circ-TTBK2 acts as a miR-217 sponge, which can inhibit HNF1 $\beta$. HNF1 $\beta$ can promote glioma malignancy.

in the process of macromolecule metabolism, membranebound organelles and protein binding. CircRNAs can also influence the immune response of viral infections through binding with immune factors, for example, NF90 and NF110 (Li et al., 2017).

\section{CNS Injury}

CircRNAs participate in various types of neuronal injury. To investigate the mechanisms of circRNAs in cerebral ischemiareperfusion-injury (IRI)-induced neuron injury, Lin et al. (2016) tested circRNA expression in HT22 cells with oxygenglucose deprivation/reoxygenation (OGD/R) and found that three circRNAs were upregulated and 12 were downregulated. It was further shown that circPTK2 could inhibit miR-29b expression. Downregulated miR-29b expression can upregulate the JAK2/STAT3 signaling pathway and finally lead to neuronal apoptosis induced by OGD-activated microglia (Wang et al., 2019). Irreversible axonal damage is the main cause of neurological dysfunction in neurodegenerative diseases or after traumatic injury. Mao et al. (2019a) found for the first time that circRNAs could be involved in axon regeneration of injured neurons. These results showed that circ-Spidr enhances axon regrowth in vitro and in vivo. In addition, the investigators found another circRNA that had the opposite effect. CircAnkib1 inhibits axon regeneration by inhibiting Schwann cell proliferation in the sciatic nerve after crush injury (Mao et al., 2019b). These studies indicated a therapeutic possibility of circRNAs for CNS injury.

\section{Retinal Disease}

As the retina is part of the CNS, many retinal diseases have also been shown to be related to circRNAs. The expression of circZRANB1 was significantly upregulated in the aqueous humor of a rat model of glaucoma. Circ-ZRANB1 is mainly derived from Müller cells, which can bind to miR-271 to regulate the expression of RUNX2. Finally, the circ-ZRANB1/miR-217/RUNX2 network influenced retinal neurodegeneration caused by Müller cells (Wang et al., 2018b). Circ-ZNF609 has a similar function in retinal neurodegeneration induced by glaucoma by binding with miR-615 (Wang et al., 2018a). In AMD patients, circNR3C1 expression was found to be downregulated in the blood serum (Chen et al., 2020a). Investigators further assessed the possible mechanism of this circRNA through RPE cell lines and found that circNR3C1 protected RPE functions via the circNR3C1miR-382-5p-PTEN network (Figure 4B). Retinoblastoma (Rb) is an important cause of blindness in early childhood (Liu et al., 2020). CircRNAs have also been shown to be dysregulated in RB and some RB cell lines. It was found that hsa_circ_0001649 was significantly downregulated and could regulate apoptosis and cell proliferation by the AKT/mTOR signaling pathway (Xing et al., 2018). There are currently few studies that have assessed the mechanism of circRNAs in retinal diseases. 


\section{Glioma}

In addition, some studies have shown the function of circRNAs in nervous system tumors. Circ-ZNF264, circPCMTD1, and circTTBK2 (Figure 4C) promote cell proliferation, migration and invasion in glioma cell lines by regulating their miRNAs (Zheng et al., 2017, 2019; Zhang et al., 2019). Circ-FBXW7 has a tumor suppressor effect in glioma cells by encoding a novel protein and is positively correlated with the overall survival rate (Yang et al., 2018). A recent study found that, apart from regulating cell growth and apoptosis, circ POSTN participated in aerobic glycolysis in glioma via the miR-361-5p/TPX2 axis (Long et al., 2020). Additionally, a recent review reported that most circRNAs function as miRNA sponges in glioma (Sun et al., 2020).

CircRNAs also play a role in psychiatric diseases such as bipolar disorder and major depressive disorder (Luykx et al., 2019; Zhang Y. et al., 2020).

Overall, the aforementioned studies indicate that circRNAs play a key role in many neurological diseases. However, the underlying mechanisms of circRNAs in many diseases have not been fully elucidated. Further study is required to elucidate how circRNAs exert their effects biologically.

\section{PERSPECTIVES}

CircRNAs are novel RNAs that are abundantly expressed in numerous organisms. Numerous studies have shown that circRNAs are enriched in the nervous system. Many of them are derived from genes related to synaptic function. Synapses are critical in information transmission and regulation of neuronal activities. CircRNAs are usually small, and many circRNAs can be detected in exosomes. They may be transported by synaptic vesicles to adjacent cells to transmit information. However, more

\section{REFERENCES}

Agarwal, V., Bell, G. W., Nam, J. W., and Bartel, D. P. (2015). Predicting effective microRNA target sites in mammalian mRNAs. eLife 4:e05005.

Ashwal-Fluss, R., Meyer, M., Pamudurti, N. R., Ivanov, A., Bartok, O., Hanan, M., et al. (2014). circRNA biogenesis competes with pre-mRNA splicing. Mol. Cell 56, 55-66. doi: 10.1016/j.molcel.2014.08.019

Barrett, S. P., and Salzman, J. (2016). Circular RNAs: analysis, expression and potential functions. Development 143, 1838-1847. doi: 10.1242/dev. 128074

Barrett, S. P., Wang, P. L., and Salzman, J. (2015). Circular RNA biogenesis can proceed through an exon-containing lariat precursor. eLife 4: e07540.

Chen, B. J., Yang, B., and Janitz, M. (2018). Region-specific expression of circular RNAs in the mouse brain. Neurosci. Lett. 666, 44-47. doi: 10.1016/j.neulet.2017. 12.022

Chen, J., Zou, Q., Lv, D., Raza, M. A., Wang, X., Li, P., et al. (2019). Comprehensive transcriptional profiling of porcine brain aging. Gene 693, 1-9. doi: 10.1016/j. gene.2019.01.019

Chen, X., Jiang, C., Sun, R., Yang, D., Liu, Q., (2020a). Circular Noncoding RNA $\mathrm{NR} 3 \mathrm{C} 1$ acts as a miR-382-5p sponge to protect RPE functions via regulating PTEN/AKT/mTOR signaling pathway. Mol. Ther. 28, 929-945. doi: 10.1016/j. ymthe.2020.01.010

Chen, X. J., Li, M. L., Wang, Y. H., Mou, H., Wu, Z., Bao, S., et al. (2020b). Abundant neural circRNA Cdrlas is not indispensable for retina maintenance. Front. Cell Dev. Biol. 8:565543. doi: 10.3389/fcell.2020.565543 evidence is required. A recent study by Xu et al. (2020) showed that circRNAs in the nucleus can regulate synaptic plasticity. This finding has encouraged more investigators to identify more mechanisms of circRNAs in the nervous system.

The rapid development of biochemical methods, such as BaseScope ISH and high-throughput circRNA sequencing analysis, provides investigators with powerful tools to investigate the exact subcellular location and interaction with other molecules. If we can determine the location of circRNAs in exact types of neurons, we may be able to distinguish more types of circRNAs and further elucidate the functions of the emerging RNAs. These studies will provide powerful guidance for clinical diagnosis and treatment.

The mechanism by which age-related circRNAs accumulate in the brain is still unclear, probably because of their circular structure. This characteristic makes them resistant to RNase R and difficult to degrade, ultimately leading to their age-related accumulation. However, recent studies have found sex-biased accumulation, indicating that this phenomenon may be caused by multiple factors.

\section{AUTHOR CONTRIBUTIONS}

M-LL and WW collected the data and drafted the manuscript. Z-BJ revised and approved the manuscript. All authors contributed to the article and approved the submitted version.

\section{FUNDING}

This study was partly supported by the Beijing Natural Science Foundation (Z200014).

Chen, X. J., Zhang, Z. C., Wang, X. Y., Zhao, H. Q., Li, M. L., Ma, Y., et al. (2020c) The circular RNome of developmental retina in mice. Mol. Ther. Nucleic Acids 19, 339-349. doi: 10.1016/j.omtn.2019.11.016

Cortes-Lopez, M., Gruner, M. R., Cooper, D. A., Gruner, H. N., Voda, A. I., van der Linden, A. M., et al. (2018). Global accumulation of circRNAs during aging in Caenorhabditis elegans. BMC Genomics 19:8. doi: 10.1186/s12864-017-4386-y

Diallo, L. H., Tatin, F., David, F., Godet, A. C., Zamora, A., Prats, A. C., et al. (2019). How are circRNAs translated by non-canonical initiation mechanisms? Biochimie 164, 45-52. doi: 10.1016/j.biochi.2019.06.015

Doxakis, E. (2010). Post-transcriptional regulation of alpha-synuclein expression by mir-7 and mir-153. J. Biol. Chem. 285, 12726-12734. doi: 10.1074/jbc.m109. 086827

Dube, U., Del-Aguila, J. L., Li, Z., Budde, J. P., Jiang, S., Hsu, S., et al. (2019). An atlas of cortical circular RNA expression in Alzheimer disease brains demonstrates clinical and pathological associations. Nat. Neurosci. 22, $1903-$ 1912. doi: 10.1038/s41593-019-0501-5

Eger, N., Schoppe, L., Schuster, S., Laufs, U., and Boeckel, J. N. (2018). Circular RNA splicing. Adv. Exp. Med. Biol. 1087, 41-52. doi: 10.1007/978-981-131426-1_4

Errichelli, L., Dini Modigliani, S., Laneve, P., Colantoni, A., Legnini, I., Capauto, D., et al. (2017). FUS affects circular RNA expression in murine embryonic stem cell-derived motor neurons. Nat. Commun. 8:14741.

Feng, Z., Zhang, L., Wang, S., and Hong, Q. (2020). Circular RNA circDLGAP4 exerts neuroprotective effects via modulating miR-134-5p/CREB pathway in Parkinson's disease. Biochem. Biophys. Res. Commun. 522, 388-394. doi: 10. 1016/j.bbrc.2019.11.102 
Ghosal, S., Das, S., Sen, R., Basak, P., and Chakrabarti, J. (2013). Circ2Traits: a comprehensive database for circular RNA potentially associated with disease and traits. Front. Genet. 4:283. doi: 10.3389/fgene.2013.00283

Gruner, H., Cortes-Lopez, M., Cooper, D. A., Bauer, M., and Miura, P. (2016). CircRNA accumulation in the aging mouse brain. Sci. Rep. 6:38907.

Guo, J. U., Agarwal, V., Guo, H., and Bartel, D. P. (2014). Expanded identification and characterization of mammalian circular RNAs. Genome Biol. 15:409.

Han, J., Gao, L., Dong, J., Bai, J., Zhang, M., and Zheng, J. (2017). The expression profile of developmental stage-dependent circular RNA in the immature rat retina. Mol. Vis. 23, 457-469.

Hansen, T. B., Jensen, T. I., Clausen, B. H., Bramsen, J. B., Finsen, B., Damgaard, C. K., et al. (2013). Natural RNA circles function as efficient microRNA sponges. Nature 495, 384-388. doi: 10.1038/nature11993

He, J., Ren, M., Li, H., Yang, L., Wang, X., and Yang, Q. (2019). Exosomal circular RNA as a biomarker platform for the early diagnosis of immune-mediated demyelinating disease. Front. Genet. 10:860. doi: 10.3389/fgene.2019.00860

Ivanov, A., Memczak, S., Wyler, E., Torti, F., Porath, H. T., Orejuela, M. R., et al. (2015). Analysis of intron sequences reveals hallmarks of circular RNA biogenesis in animals. Cell Rep. 10, 170-177. doi: 10.1016/j.celrep.2014.12.019

Jeck, W. R., Sorrentino, J. A., Wang, K., Slevin, M. K., Burd, C. E., Liu, J., et al. (2013). Circular RNAs are abundant, conserved, and associated with ALU repeats. RNA 19, 141-157. doi: 10.1261/rna.035667.112

Jin, Z. B., Gao, M. L., Deng, W. L., Wu, K. C., Sugita, S., Mandai, M., et al. (2019). Stemming retinal regeneration with pluripotent stem cells. Prog. Retin. Eye Res. 69, 38-56. doi: 10.1016/j.preteyeres.2018.11.003

Kelly, S., Greenman, C., Cook, P. R., and Papantonis, A. (2015). Exon skipping is correlated with exon circularization. J. Mol. Biol. 427, 2414-2417. doi: 10.1016/ j.jmb.2015.02.018

Knupp, D., and Miura, P. (2018). CircRNA accumulation: A new hallmark of aging? Mech. Ageing Dev. 173, 71-79. doi: 10.1016/j.mad.2018.05.001

Legnini, I., Di Timoteo, G., Rossi, F., Morlando, M., Briganti, F., Sthandier, O., et al. (2017). Circ-ZNF609 is a circular rna that can be translated and functions in myogenesis. Mol. Cell 66, 22-37.e9.

Li, X., Liu, C. X., Xue, W., Zhang, Y., Jiang, S., Yin, Q. F., et al. (2017). Coordinated circRNA biogenesis and function with NF90/NF110 in viral infection. Mol. Cell 67, 214-227.e7.

Li, Z., Huang, C., Bao, C., Chen, L., Lin, M., Wang, X., et al. (2015). Exon-intron circular RNAs regulate transcription in the nucleus. Nat. Struct. Mol. Biol. 22, 256-264. doi: 10.1038/nsmb.2959

Lin, S. P., Ye, S., Long, Y., Fan, Y., Mao, H. F., Chen, M. T., et al. (2016). Circular RNA expression alterations are involved in OGD/R-induced neuron injury. Biochem. Biophys. Res. Commun. 471, 52-56. doi: 10.1016/j.bbrc.2016.01.183

Liu, H., Zhang, Y., Zhang, Y. Y., Li, Y. P., Hua, Z. Q., Zhang, C. J., et al. (2020). Human embryonic stem cell-derived organoid retinoblastoma reveals a cancerous origin. Proc. Natl. Acad. Sci. U.S.A. 117, 33628-33638. doi: 10.1073/ pnas. 2011780117

Long, N., Chu, L., Jia, J., Peng, S., Gao, Y., Yang, H., et al. (2020). CircPOSTN/miR361-5p/TPX2 axis regulates cell growth, apoptosis and aerobic glycolysis in glioma cells. Cancer Cell Int. 20:374.

Lukiw, W. J., and Circular, R. N. A. (2013). (circRNA) in Alzheimer's disease (AD). Front. Genet. 4:307. doi: 10.3389/fgene.2013.00307

Luykx, J. J., Giuliani, F., Giuliani, G., and Veldink, J. (2019). Coding and noncoding RNA abnormalities in bipolar disorder. Genes 10:946. doi: 10.3390/ genes10110946

Mao, S., Huang, T., Chen, Y., Shen, L., Zhou, S., Zhang, S., et al. (2019a). CircSpidr enhances axon regeneration after peripheral nerve injury. Cell Death Dis. 10:787.

Mao, S., Zhang, S., Zhou, S., Huang, T., Feng, W., Gu, X., et al. (2019b). A Schwann cell-enriched circular RNA circ-Ankib1 regulates Schwann cell proliferation following peripheral nerve injury. FASEB J. 33, 12409-12424. doi: 10.1096/fj. $201900965 \mathrm{r}$

Memczak, S., Jens, M., Elefsinioti, A., Torti, F., Krueger, J., Rybak, A., et al. (2013). Circular RNAs are a large class of animal RNAs with regulatory potency. Nature 495, 333-338. doi: 10.1038/nature 11928

Pamudurti, N. R., Bartok, O., Jens, M., Ashwal-Fluss, R., Stottmeister, C., Ruhe, L., et al. (2017). Translation of CircRNAs. Mol. Cell 66, 9-21.e7.

Petkovic, S., and Muller, S. (2015). RNA circularization strategies in vivo and in vitro. Nucleic Acids Res. 43, 2454-2465. doi: 10.1093/nar/gkv045
Piwecka, M., Glazar, P., Hernandez-Miranda, L. R., Memczak, S., Wolf, S. A., Rybak-Wolf, A., et al. (2017). Loss of a mammalian circular RNA locus causes miRNA deregulation and affects brain function. Science 357:eaam8526. doi: $10.1126 /$ science.aam 8526

Rybak-Wolf, A., Stottmeister, C., Glazar, P., Jens, M., Pino, N., Giusti, S., et al. (2015). Circular RNAs in the mammalian brain are highly abundant, conserved, and dynamically expressed. Mol. Cell 58, 870-885. doi: 10.1016/j.molcel.2015. 03.027

Salzman, J., Chen, R. E., Olsen, M. N., Wang, P. L., and Brown, P. O. (2013). Cell-type specific features of circular RNA expression. PLoS Genet. 9:e1003777. doi: 10.1371/journal.pgen.1003777

Sanger, H. L., Klotz, G., Riesner, D., Gross, H. J., and Kleinschmidt, A. K. (1976). Viroids are single-stranded covalently closed circular RNA molecules existing as highly base-paired rod-like structures. Proc. Natl. Acad. Sci. U.S.A. 73, 3852-3856. doi: 10.1073/pnas.73.11.3852

Shi, Z., Chen, T., Yao, Q., Zheng, L., Zhang, Z., Wang, J., et al. (2017). The circular RNA ciRS-7 promotes APP and BACE1 degradation in an NF-kappaB-dependent manner. FEBS J. 284, 1096-1109. doi: 10.1111/febs. 14045

Sun, J., Li, B., Shu, C., Ma, Q., and Wang, J. (2020). Functions and clinical significance of circular RNAs in glioma. Mol. Cancer 19:34.

Sun, L. F., Zhang, B., Chen, X. J., Wang, X. Y., Zhang, B. W., Ji, Y. Y., et al. (2019). Circular RNAs in human and vertebrate neural retinas. RNA Biol. 16, 821-829. doi: 10.1080/15476286.2019.1591034

Suzuki, H., Zuo, Y., Wang, J., Zhang, M. Q., Malhotra, A., and Mayeda, A. (2006). Characterization of RNase R-digested cellular RNA source that consists of lariat and circular RNAs from pre-mRNA splicing. Nucleic Acids Res. 34:e63. doi: 10.1093/nar/gkl151

Venø, M. T., Hansen, T. B., Venø, S. T., Clausen, B. H., Grebing, M., Finsen, B., et al. (2015). Spatio-temporal regulation of circular RNA expression during porcine embryonic brain development. Genome Biol. 16:245.

Wang, H., Li, Z., Gao, J., and Liao, Q. (2019). Circular RNA circPTK2 regulates oxygen-glucose deprivation-activated microglia-induced hippocampal neuronal apoptosis via miR-29b-SOCS-1-JAK2/STAT3-IL-1beta signaling. Int. J. Biol. Macromol. 129, 488-496. doi: 10.1016/j.ijbiomac.2019. 02.041

Wang, J. J., Liu, C., Shan, K., Liu, B. H., Li, X. M., Zhang, S. J., et al. (2018a). Circular RNA-ZNF609 regulates retinal neurodegeneration by acting as miR-615 sponge. Theranostics 8, 3408-3415. doi: 10.7150/thno. 25156

Wang, J. J., Shan, K., Liu, B. H., Liu, C., Zhou, R. M., Li, X. M., et al. (2018b). Targeting circular RNA-ZRANB1 for therapeutic intervention in retinal neurodegeneration. Cell Death Dis. 9:540.

Westholm, J. O., Miura, P., Olson, S., Shenker, S., Joseph, B., Sanfilippo, P., et al. (2014). Genome-wide analysis of drosophila circular RNAs reveals their structural and sequence properties and age-dependent neural accumulation. Cell Rep. 9, 1966-1980. doi: 10.1016/j.celrep.2014. 10.062

Xia, S., Feng, J., Lei, L., Hu, J., Xia, L., Wang, J., et al. (2017). Comprehensive characterization of tissue-specific circular RNAs in the human and mouse genomes. Brief. Bioinform. 18, 984-992.

Xing, L., Zhang, L., Feng, Y., Cui, Z., and Ding, L. (2018). Downregulation of circular RNA hsa_circ_0001649 indicates poor prognosis for retinoblastoma and regulates cell proliferation and apoptosis via AKT/mTOR signaling pathway. Biomed. Pharmacother. 105, 326-333. doi: 10.1016/j.biopha.2018.05. 141

Xu, K., Zhang, Y., Xiong, W., Zhang, Z., Wang, Z., Lv, L., et al. (2020). CircGRIA1 shows an age-related increase in male macaque brain and regulates synaptic plasticity and synaptogenesis. Nat. Commun. 11:3594.

Yang, H., Wang, H., Shang, H., Chen, X., Yang, S., Qu, Y., et al. (2019). Circular RNA circ_0000950 promotes neuron apoptosis, suppresses neurite outgrowth and elevates inflammatory cytokines levels via directly sponging miR-103 in Alzheimer's disease. Cell Cycle 18, 2197-2214. doi: 10.1080/15384101.2019. 1629773

Yang, Y., Gao, X., Zhang, M., Yan, S., Sun, C., Xiao, F., et al. (2018). Novel role of FBXW7 circular RNA in repressing glioma tumorigenesis. J. Natl. Cancer Inst. 110, 304-315. doi: 10.1093/jnci/djx166 
You, X., Vlatkovic, I., Babic, A., Will, T., Epstein, I., Tushev, G., et al. (2015). Neural circular RNAs are derived from synaptic genes and regulated by development and plasticity. Nat. Neurosci. 18, 603-610. doi: 10.1038/nn.3975

Zaphiropoulos, P. G. (1997). Exon skipping and circular RNA formation in transcripts of the human cytochrome P-450 2C18 gene in epidermis and of the rat androgen binding protein gene in testis. Mol. Cell. Biol. 17, 2985-2993. doi: $10.1128 / \mathrm{mcb} \cdot 17.6 .2985$

Zhang, F., Mai, S. R., and Zhang, L. (2019). Circ-ZNF264 promotes the growth of glioma cells by upregulating the expression of miR-4493 target gene Apelin. J. Mol. Neurosci. 69, 75-82. doi: 10.1007/s12031-019-01334-8

Zhang, N., Gao, Y., Yu, S., Sun, X., and Shen, K. (2020). Berberine attenuates Abeta42-induced neuronal damage through regulating circHDAC9/miR-1425p axis in human neuronal cells. Life Sci. 252:117637. doi: 10.1016/j.lfs.2020. 117637

Zhang, Y., Du, L., Bai, Y., Han, B., He, C., Gong, L., et al. (2020). CircDYM ameliorates depressive-like behavior by targeting miR-9 to regulate microglial activation via HSP90 ubiquitination. Mol. Psychiatry 25, 1175-1190. doi: 10 . 1038/s41380-018-0285-0

Zhang, Y., Xue, W., Li, X., Zhang, J., Chen, S., Zhang, J. L., et al. (2016). The biogenesis of nascent circular RNAs. Cell Rep. 15, 611-624. doi: 10.1016/j. celrep.2016.03.058

Zhang, Y., Zhang, X. O., Chen, T., Xiang, J. F., Yin, Q. F., Xing, Y. H., et al. (2013). Circular intronic long noncoding RNAs. Mol. Cell 51, 792-806. doi: 10.1016/j.molcel.2013.08.017
Zhao, Y., Alexandrov, P. N., Jaber, V., and Lukiw, W. J. (2016). Deficiency in the Ubiquitin Conjugating Enzyme UBE2A in Alzheimer's Disease (AD) is Linked to Deficits in a Natural Circular miRNA7 Sponge (circRNA; ciRS-7). Genes 7:116. doi: 10.3390/genes712 0116

Zheng, J., Liu, X., Xue, Y., Gong, W., Ma, J., Xi, Z., et al. (2017). TTBK2 circular RNA promotes glioma malignancy by regulating miR-217/HNF1beta/Derlin-1 pathway. J. Hematol. Oncol. 10:52.

Zheng, S. Q., Qi, Y., Wu, J., Zhou, F. L., Yu, H., Li, L., et al. (2019). CircPCMTD1 acts as the sponge of miR-224-5p to promote Glioma progression. Front. Oncol. 9:398. doi: 10.3389/fonc.2019.00398

Zhou, B., Yang, H., Yang, C., Bao, Y. L., Yang, S. M., Liu, J., et al. (2021). Translation of noncoding RNAs and cancer. Cancer Lett. 497, 89-99.

Conflict of Interest: The authors declare that the research was conducted in the absence of any commercial or financial relationships that could be construed as a potential conflict of interest.

Copyright (c) $2021 \mathrm{Li}$, Wang and Jin. This is an open-access article distributed under the terms of the Creative Commons Attribution License (CC BY). The use, distribution or reproduction in other forums is permitted, provided the original author(s) and the copyright owner(s) are credited and that the original publication in this journal is cited, in accordance with accepted academic practice. No use, distribution or reproduction is permitted which does not comply with these terms. 